\title{
高等学校化学类专业物理化学实验教学内容与教学要求建议
}

张树永 ${ }^{1,}{ }^{*}$, 范楼珍 ${ }^{2}$, 淳远 ${ }^{3}$, 刘永梅 ${ }^{4}$, 田福平 ${ }^{5}$, 白云山 ${ }^{6}$, 宋淑娥 ${ }^{1}$

1 山东大学化学与化工学院, 济南 250100

2 北京师范大学化学学院, 北京 100875

3 南京大学化学化工学院, 南京 210023

4 复旦大学化学系, 上海 200433

5 大连理工大学化工学院, 辽宁大连 116024

6 陕西师范大学化学化工学院, 西安 710062

摘要: 为了推进化学类专业本科教学质量国家标准和教育部化学类专业教学指导委员会相关要求的全面落实, 推进 物理化学实验的改革与建设, 本建议明确了化学类专业物理化学实验的教学目标和相关教学要求, 通过示例明确了 物理化学参数或者性质的测量原理和仪器设备, 明确相关教学实验所应达到的知识、能力与素质目标。对物理化学 实验改革与建设具有指导意义。

关键词: 化学类专业; 物理化学实验; 教学内容; 教学要求; 建议

中图分类号: G64; O6

\section{Suggestions on Teaching Contents and Requirements of Physical Chemistry Laboratory for Chemistry Majors of Higher Chemistry Education}

\author{
Shuyong Zhang ${ }^{1, *}$, Louzhen Fan ${ }^{2}$, Yuan Chun ${ }^{3}$, Yongmei Liu ${ }^{4}$, Fuping Tian ${ }^{5}$, Yunshan Bai ${ }^{6}$, \\ Shue Song ${ }^{1}$ \\ ${ }^{1}$ School of Chemistry and Chemical Engineering, Shandong University, Jinan 250100, China. \\ ${ }^{2}$ College of Chemistry, Beijing Normal University, Beijing 100875, China. \\ ${ }^{3}$ School of Chemistry and Chemical Engineering, Nanjing University, Nanjing 210023, China. \\ ${ }^{4}$ Department of Chemistry, Fudan University, Shanghai 200433, China. \\ ${ }^{5}$ School of Chemical Engineering, Dalian University of Technology, Dalian 116024, Liaoning Province, China. \\ ${ }^{6}$ School of Chemistry and Chemical Engineering, Shaanxi Normal University, Xi'an 710062, China.
}

\begin{abstract}
To implement the National Teaching Standard for Chemistry Majors and related requirements proposed by the Instructional Committee of Higher Chemistry Education, Ministry of Education, suggestions of physical chemistry laboratory teaching are made. The teaching goals and contents of the overall and definite physical chemistry laboratory are both defined. The principles for measuring physicochemical parameters and properties are clarified based on some typical experiments with special devices. The laboratory teaching goals including knowledge, ability and quality that should be achieved are suggested. It is of guiding significance for the further reform and construction of physical chemistry laboratory.
\end{abstract}


Key Words: Chemistry majors; Physical chemistry lab; Teaching contents; Teaching requirements; Suggestions

\section{1 引言}

化学是一门理论与实验并重、富有创造性的中心学科 ${ }^{[1]}$, 实验教学在化学类专业人才培养中占 据不可或缺的地位。相对于其他实验, 物理化学实验原理复杂综合、使用仪器设备多种多样、测量 次数多周期长、数据处理和分析要求高 ${ }^{[2]}$, 在培养学生实验思维和科学素养方面发挥着重要作用。

《普通高等学校本科专业类教学质量国家标准》 ${ }^{[1]}$ 和《化学类专业化学实验教学建议内容》 ${ }^{[3]}$ 仅列出 了实验教学知识点, 未明确实验能力和素质培养要求 ${ }^{[4]}$ 。受教育部高等学校化学类专业教学指导委 员会委托和立项支持, 由山东大学张树永教授牵头, 联合北京师范大学范楼珍教授、南京大学淳远 教授、复旦大学刘永梅副教授、大连理工大学田福平副教授、陕西师范大学白云山教授、山东大学 宋淑娥高级工程师组成课题组开展了本建议的研制工作。

本建议是指导性的, 它既非物理化学实验教学的最高要求也不是最低要求, 所列内容也非必须 在物理化学实验中开设。各高校可以在建议的基础上根据自身办学定位, 结合专业特色和学生就业 发展需要增减相关内容, 构建各具特色的实验教学内容和教学体系, 满足人才培养需要。

\section{2 物理化学实验能力和素质培养要求}

实验教学可分为四个层次: 一是学科知识层次, 包括事实性知识和概念性知识, 要求学生说明 相关概念、原理和注意事项等; 二是具体方法层次, 属于程序性知识, 学生能够按照规程实际操作 仪器设备, 完成实验; 三是方法综合层次, 学生能够根据任务要求, 综合设计并完成实验, 对实验 结果进行分析讨论, 得出有效结论; 四是方法创新层次, 相当于元认知 ${ }^{[5,6]}$, 学生能够领悟实验本质, 改进或者创新测量的原理和方法并进行应用拓展。其中, 第一、二层次是实验教学的基本要求, 本 科教学应当达到第三层次并努力达到第四层次, 实现以下目标 ${ }^{[2-4]}$ :

（1）能够阐述相关物理量和物理化学参数的定义及其物理含义;

（2）说明相关测量的原理和方法, 对不同原理和方法进行比较, 说明其优势与不足; 能够合理 选择测量的原理和方法, 有效减少系统误差;

(3) 能够设计并评价测量方案。能够根据系统、测量时间和精度要求等, 选择适宜的测量原理 和仪器设备, 设计实验方案; 能够从法律、安全和环保等多个角度对方案进行分析评价, 提出优化 的思路和方法, 明确方案的可行性和可能存在的问题, 明确个人的责任和义务; 能够在方案设计中 体现创新性;

（4）能够搭建和操作实验装置。能够根据实验方案独立搭建实验装置; 能够设计制作必要的实 验装置; 能够对仪器设备或实验系统进行检查和校准; 能够规范操作仪器和系统完成实验;

(5) 能够规范观察和记录。能够全面细致观察实验过程, 规范记录实验现象和实验数据;

(6) 能够科学分析数据。能够判断数据的合理性, 正确取舍数据; 能够对实验数据进行处理, 得 出相关规律和合理结论; 能够利用信息技术展示数据和结论;

(7) 能够分析和解决问题。能够分析解决实验过程中发现或者发生的问题, 保证实验顺利进行。 说明测量的影响因素, 并通过方案优化和数据处理, 保证测量的准确度和精密度, 减少偶然误差; 能够对实验现象和规律进行解释;

(8) 能够联系实际, 说明相关实验在科学研究和生产生活中的拓展应用;

(9) 能够自主查阅资料, 发现并借鉴相关测量原理和方法的最新进展, 提出改进测量装置和方 法的建议。

在实验过程中还应养成科学精神、科学素养和研究能力, 养成规范严谨的实验习惯、实事求是 的科学态度、分工协作的团队精神、坚韧不拔的意志品质、勇于探索的创新精神 ${ }^{[2]}$ 。 


\section{3 物理化学实验内容和教学要求建议}

\section{1 建议内容概述}

本建议的编制, 首先根据《化学类专业化学实验教学建议内容》 “III基本物理量、物理化学参数 与性质的测量” [3]确定需要测量的物理参数或者性质, 而后结合需要掌握的仪器及测量原理, 参考 国内重要实验教材 ${ }^{[7-32]}$, 列举典型实验, 并在此基础上明确实验所需达成的知识、能力和素质目标。 建议内容分为: (I) 基本物理化学参数测量; (II) 热力学性质; (III) 动力学性质; (IV) 电化学性质; (V) 表面与胶体性质; (VI) 分子的结构与性质共6个部分。各部分所包含的参数和性质可参考文后附 图。在选择实验原理和方法时, 各高校可根据自身条件和特色, 选择 $1-2$ 个即可, 其他原理和方法可 作为学生设计实验或者教师更新实验的参考, 也可作为研讨课内容。建议中带 “*” 的内容为拓展内 容或与科研对接的内容, 仅供参考。

\section{2 具体内容与建议}

\section{(I) 基本物理化学参数的测量}

\section{(I-1) 气体常数}

典型实验: 摩尔气体常数的测定 ${ }^{[7]}$

仪器设备: 电子天平 温度计 气压计 具有刻度的密闭容器

实验建议: (1) 说明利用 $(p V / n)-T$ 作图确定气体常数的原理; (2) 能够设计其他测量气体常数的 实验; (3) 说明测量玻尔兹曼常数的原理。

\section{(I-2) 阿伏伽德罗常数}

典型实验: 阿伏伽德罗常数的测定 ${ }^{[7]}$

仪器设备：电子天平 气量管(碱式滴定管) 气压计 温度计 直流电源 电量计

实验建议: (1) 能够说明阿伏伽德罗常数的定义; (2) 能够说明通过置换反应、电解法、布朗运 动法、*X射线衍射法、*黑体辐射法、*光散射法、*油滴法、*单分子膜测量法等测量阿伏伽德罗常 数的原理; (3) 能够对主要测量原理和方法进行比较。

\section{(I-3) 浓度(含量)}

典型实验: 比色法研究甲基紫反应动力学 ${ }^{[8]}$; 蔗糖水解速率常数的测定 ${ }^{[9]}$; 乙酸乙酯㿝化反应速 率常数的测定 ${ }^{[9]}$; 氯离子选择性电极的测定及其应用 ${ }^{[11]}$

仪器设备：滴定管 分光光度计 旋光仪 电导率仪 阿贝折射仪 离子计 *光谱仪

实验建议: (1) 能够说明采用滴定法(酸碱滴定、配合滴定、氧化还原滴定、沉淀滴定)、重量分 析法、分光光度法、旋光度法、折射率法、*浓度计、*浓度(含量)传感器等测量浓度(含量)的原理和 方法; (2) 能够说明采用物理方法测量浓度(含量)的优势及局限性; (3) 能够分析提高测量准确度的 方法; (4) 能够说明采用离子选择性电极测量离子浓度的原理。

\section{(I-4) pH}

典型实验：电动势的测定及其应用 ${ }^{[9]}$; 电势 $\mathrm{pH}$ 曲线的测定 ${ }^{[12]}$

仪器设备: $\mathrm{pH}$ 计 玻璃电极 氢电极 $*$ 其他响应电极

实验建议: (1) 能够说明采用酸碱指示剂、 $\mathrm{pH}$ 试纸、 $\mathrm{pH}$ 计测量 $\mathrm{pH}$ 的原理; (2) 能够说明 $\mathrm{pH}$ 指示 电极(玻璃电极、氢电极)的响应原理; (3) 能够进行 $\mathrm{pH}$ 计校正; (4) 能够说明 $\mathrm{pH}$ 测量的可操作定义; (5) * 能够说明Harned法测量 $\mathrm{pH}$ 的原理。

\section{(I-5) 溶解度/溶度积}

典型实验: 光度法测定碘酸铜的溶度积常数 ${ }^{[7]}$; 电动势的测定及其应用 ${ }^{[9]}$; 电导法测定难溶盐的 溶解度 ${ }^{[13]}$; 电池电动势法测定氯化银的溶度积 ${ }^{[13]}$

仪器设备: 电子天平 气相色谱仪 液相色谱仪 分光光度计 电位差计 电导率仪 溶解度 测量仪 
实验建议: (1) 能够说明采用平衡法(化学滴定法、重量法、色谱法、分光光度法、电导法、电 动势法)和*动态法(析晶法)测量溶解度的原理; (2) 能够说明采用电导法测量难溶盐溶解度的原理; (3) 能够说明采用电势法测量难溶盐溶度积的原理。

\section{(I-6) 密度}

典型实验: (乙醇-水/ $\mathrm{NaCl}$-水) 溶液偏摩尔体积的测 ${ }^{\text {定 }}{ }^{[8,11]}$; 溶液法测定极性分子的偶极矩 ${ }^{[15]}$; 密 度法测定聚合物结晶度 ${ }^{[14]}$

仪器设备: 密度计(浮力式密度计、*静压式密度计、*振动式密度计、*声速式密度计、*重力式 密度计) 密度管(比重管) 密度瓶(比重瓶) 密度天平 密度梯度柱

实验建议: (1) 能够说明质量体积法(密度计法/比重计法、密度瓶法)、浮力法、密度梯度法、 *落滴法、*压强法、*振动管法、*放射性同位素法、*声速法测量密度的原理; (2) 能够对密度瓶进 行校准; (3) 能够说明密度梯度法测量固体密度的原理。

\section{(I-7) 熔点}

典型实验：熔点测定与温度计校正 ${ }^{[16]}$

仪器设备: 毛细管 提勒(Thiele)管 数字熔点测定仪 显微熔点测定仪 *自动视频熔点仪 *金属相图仪

实验建议: (1) 能够说明等压下纯物质的熔点为定值的原因; (2) 能够说明各类方法的测量原理;

(3) 能够说明熔点测量的影响因素及其用途。

\section{(I-8) 沸点}

典型实验：双液体系沸点-组成图的绘制[8]

仪器设备：沸点仪 阿贝折射仪 提勒管 沸点测定仪

实验建议: (1) 能够说明常量法、微量法测量沸点的原理; (2) 能够说明等压下纯物质沸点恒定 的原因; (3) 能够说明沸点测量的影响因素和减小误差的方法。

\section{(I-9) (饱和)蒸气压}

典型实验：液体饱和蒸气压的测定一一静态法 ${ }^{[9]}$

仪器设备: 蒸气压测定装置 气相色谱

实验建议: (1) 能够说明动态法、静态法、饱和气流法、气相色谱法测量饱和蒸气压的原理和方 法; (2) 能够结合克劳修斯-克拉贝龙方程、气液平衡、理想气体方程、拉乌尔定律等说明蒸气压与 温度的关系；(3) 能够说明外压与液体饱和蒸气压的关系；(4) 能够说明相对湿度的概念。

\section{(I-10) 黏度(同I-16)}

典型实验：黏度法测高聚物的相对分子质量 ${ }^{[9,15]}$; 无机盐混合溶液黏度的测定 ${ }^{[12]}$

仪器设备：乌氏黏度计 奥氏黏度计

实验建议: (1) 能够辨析特性黏度、比浓黏度、增比黏度等相关概念; (2) 能够说明毛细管黏度 法、*恩氏黏度法的测量原理; (3) 能够测量聚合物的黏均分子量并说明其影响因素。

\section{(I-11) 折射率}

典型实验：乙醇-环己烷双液系相图的绘制 ${ }^{[13]}$; 摩尔折射度的测定 ${ }^{[15]}$; 挥发性二组分系统 $T-x$ 图 的绘制[15]

\section{仪器设备: 阿贝折射仪}

实验建议: (1) 能够说明阿贝折射仪的工作原理；(2) 能够说明阿贝折射仪法、掠面入射法、*最 小偏角法、*棱镜耦合法、*反射光谱法、*双光干涉法、干涉显微镜、*光干涉仪等测量折射率的原 理并进行对比; (3) 能够说明通过折射率仪测量浓度的原理。 


\section{(I-12) 比旋光度}

典型实验：旋光度的测定 ${ }^{[16]}$; 蔗糖水解反应速率常数的测定 ${ }^{[10]}$

仪器设备: 旋光仪

实验建议：(1) 能够说明旋光性和旋光度等概念；(2) 能够说明旋光仪的工作原理；(3) 能够通 过旋光度测量浓度并说明其原理。

\section{(I-13) 消光系数/摩尔吸光系数}

典型实验: 吸光度的测定 ${ }^{[12,15]}$

仪器设备: 分光光度计

实验建议：(1) 能够说明朗伯-比尔定律及其应用前提; (2) 能够说明测量消光系数/摩尔吸光系 数的意义。

\section{(I-14) 玻璃化转变温度 $\left(T_{\mathrm{g}}\right)$}

典型实验: 示差扫描量热测定玻璃化转变温度 ${ }^{[17]}$

仪器设备: 示差扫描量热仪

实验建议: (1) 能够说明 $T_{\mathrm{g}}$ 的物理意义; (2) 能够说明采用微分扫描示差量热法(DSC)、差热分 析法(DTA)、*比容法、*折射法、*核磁共振波谱法测量 $T_{\mathrm{g}}$ 的原理; (3) 能够说明 $T_{\mathrm{g}}$ 对材料性能的影响。

\section{(I-15) 相对分子质量}

\section{(I-15-1) 气体的相对分子量}

典型实验: 二氧化碳相对分子量的测定 ${ }^{[18]}$

仪器设备: 电子天平 锥形瓶

实验建议: (1) 能够能够进行气体的制备与净化; (2) 能够说明测量气体物质分子量的方法及 其原理; (3) 能够分析影响测量精度的因素。

\section{(I-15-2) 其他物质相对分子量}

典型实验：凝固点降低法测相对分子质量 ${ }^{[9]}$; 凝固点降低法测定萎的相对分子质量 ${ }^{[7]}$

仪器设备: 凝固点测定仪 精密温度/温差测量仪

实验建议: (1) 能够说明凝固点降低法、沸点升高法、渗透压法、*扩散速率法、*超离心法等方 法测量分子量的原理; (2) 能够正确使用冰水浴或冰盐浴, 并根据不同的待测物选择适宜的溶剂和 冷却介质；(3) 能够说明研究分子解离或缔合现象的原理；(4) 能够校正过冷现象的影响。

\section{(I-16) 高分子相对分子质量及其分布}

典型实验：黏度法测高聚物的相对分子质量 ${ }^{[8,9]}$

仪器设备: 乌氏黏度计

实验建议: (1) 同(I-10); (2) 能够说明黏度法、渗透压法、唐南平衡法、凝胶渗透色谱法、*光 散射法、*超离心法测量分子量的原理; (3) 能够采用渗透压计、凝胶渗透色谱、*光散射光度计进行 测量; (4) 能够比较不同测试原理和方法的适用范围; (5) 能够说明高分子的相对分子质量及其分布 对材料性能的影响。

\section{(I-17) 偏摩尔量}

\section{(I-17-1) 偏摩尔体积}

典型实验：乙醇-水/ $\mathrm{NaCl}-$ 水体系偏摩尔体积的测定 ${ }^{[8,11]}$

仪器设备: 密度瓶 恒温水浴 电子分析天平 密度计

实验建议: 说明多组分系统的热力学性质与其组成的关系。

\section{(I-17-2) 偏摩尔混合热}

典型实验：色谱法测定无限稀溶液的活度系数 ${ }^{[8]}$; 活度系数的测 ${ }^{\text {定 }}{ }^{[11]}$

仪器设备: 气相色谱仪 氢气发生器 微量注射器 流量计 
实验建议: 说明气相色谱法测量偏摩尔混合热的原理。

\section{(I-18) 电离度(电离平衡常数)}

典型实验: 醋酸解离常数的测定 ${ }^{[7]}$; 电导法测定弱电解质的电离平衡常数 ${ }^{[10]}$; 用分光光度法测 定弱电解质的解离常数 ${ }^{[9]}$; 核磁共振法测定水溶液中反应的平衡常数及反应速率常数 ${ }^{[9]}$

仪器设备: 电导率仪 分光光度计 核磁共振谱仪

实验建议: 能够说明电导法、 $\mathrm{pH}$ 法、分光光度法、核磁共振法等测量平衡常数的原理。

\section{(I-19) 分配系数}

典型实验: 化学反应平衡常数及分配系数的测定 ${ }^{[8]}$; 吸光度法测量水杨酸在水和氯仿中的分配 系数 ${ }^{[8]}$; 分配系数测定 ${ }^{[19]}$; 药物溶解度与分配系数的测定 ${ }^{[20]}$

仪器设备: 分光光度计

实验建议: (1) 能够说明分配系数测量对物质分离操作的重要意义; (2) 能够说明影响分配系 数的因素; (3) 能够说明分配系数测定原理与方法; (4) 能够说明萃取操作的重要应用; (5) 能够说 明在气相色谱和液相色谱中物质分离的原理。

\section{(I-20) 活度系数}

典型实验：色谱法测定无限稀溶液的活度系数 ${ }^{[8]}$; 气相色谱法测定无限稀释溶液的活度系数 ${ }^{[9]}$; 用紫外分光光度计测定荎在硫酸铵水溶液中的活度系数 ${ }^{[11]}$; 冰点下降法测定正十六烷中TBP的活度 系数 ${ }^{[21]}$; 电解质溶液活度系数的测定 ${ }^{[22]}$

仪器设备: 凝固点测定仪 电势差计 分光光度计 气相色谱仪

实验建议: (1) 能够说明通过凝固点降低、蒸气压、熔点降低、沸点升高、渗透压、电动势、 色谱等方法测量活度系数的原理; (2) 能够说明电解质和非电解质溶液活度系数随浓度变化的不同 及原因; (3) 能够说明消除活度对有离子参与反应影响的原理。

\section{(II-1) 热效应}

\section{(II) 热力学性质}

热效应主要包括燃烧热、反应热、溶解热、*稀释热、吸附热、*升华热、*熔融热、*气化热等。

\section{(II-1-1) 燃烧热}

典型实验：燃烧热的测定 ${ }^{[8-13]}$

仪器设备: 氧弹式量热计

实验建议: (1) 能够说明基准物质选择的原理, 能够绘制雷诺校正图并对数据进行校正; (2) 能 够分析导致燃烧热测量误差的原因并进行优化; (3) 能够说明通过可逆电池测量燃烧热的原理; (4) 能 够对固体样品和液体样品的燃烧热进行测量; (5) 能够对实验进行绿色化、实用化改造, 如测定蔗 糖、食品、生物质、煤炭等的燃烧热; (6) 能够说明燃烧热测量的意义。

\section{(II-1-2) 溶解热和反应热}

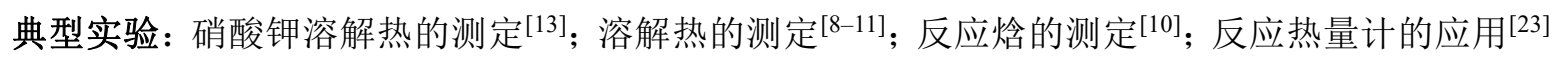

仪器设备: 反应量热器(杜瓦瓶) 温度计(感温元件/测温仪) 恒流源 加热器 反应管

实验建议: (1) 能够说明采用绝热反应装置测量反应热的原理, 能够采用电热补偿法进行测定; (2) 能够说明温差校正的方法; (3) 能够说明影响测量的因素; (4) 能够将测量原理推广到中和热、稀 释热等的测量; (5) 能够采用作图法求稀释热。

\section{*(II-1-3) 相变热}

典型实验: 聚对苯二甲酸乙二醇酯示差扫描量热分析 ${ }^{[24]}$

仪器设备: 微分示差扫描量热计 
实验建议: (1) 能够说明示差扫描量热法测量相变或化学反应热效应的原理; (2) 能够使用示差 扫描量热法测量聚合物的玻璃化转变温度、熔融温度及相关热效应; (3) 能够说明相变储能材料的 储能原理。

\section{*(II-1-4) 吸附热}

典型实验：气相色谱法测定催化剂吸附热[25]

仪器设备: 气相色谱仪 氮气钢瓶 氮气表 稳压器 皇膜流量计

实验建议: (1) 能够说明测量吸附热的意义; (2) 能够说明气相色谱仪测定吸附热的原理。

\section{*(II-1-5) 气化热}

典型实验：饱和蒸气压法测定乙醇的气化热[26]

仪器设备：液体蒸气压测定装置 数字式温度计

实验建议：能够说明通过测量蒸气压随温度的变化测量物质气化热的原理。

(II-2) 平衡常数

\section{(II-2-1) 气/固相反应平衡常数}

典型实验: 氨基甲酸铵分解反应平衡常数的测定 ${ }^{[8,11]}$; 气相反应平衡常数的测定 ${ }^{[9]}$; 合成氨反应 平衡常数的测定 ${ }^{[27]}$

仪器设备：压力计 等压计 缓冲瓶 真空原 低真空测压仪 合成氨实验装置

实验建议: (1) 能够说明反应平衡常数与压力、温度及热力学函数的关系; (2) 能够说明测量各 类反应平衡常数的原理和注意事项。

\section{(II-2-2) 液相反应平衡常数}

典型实验: 核磁共振法测定质子化反应的平衡常数 ${ }^{[9]}$; 分光光度法测定甲基红电离平衡常数 ${ }^{[11]}$; 碘和碘离子反应平衡常数的测定 ${ }^{[14]}$; 电导法测定弱电解质的解离平衡常数 ${ }^{[8]}$; 醋酸解离度和解离常 数的测定 ${ }^{[18]}$

仪器设备: 分光光度计 电导率仪 $\mathrm{pH}$ 计 核磁共振谱仪

实验建议: (1) 能够说明分光光度法、电导法、核磁共振法、pH计法测定平衡常数的原理; (2) 能 够说明分光光度法测量混合组分浓度的原理。

\section{(II-2-3) 配合物(不)稳定常数和配位数(同VI-3)}

典型实验：配合物组成及不稳定常数的测 ${ }^{\text {定 }}{ }^{[13]}$; 磺基水杨酸合铁配合物组成和稳定常数的测定 ${ }^{[8]}$

仪器设备: 分光光度计

实验建议: (1) 能够说明用分光光度法测量配合物稳定常数的原理及适用条件; (2) 能够列举其 他测量配合物稳定常数的方法并进行比较。

(II-3) 相图

典型相图包括: 沸点-组成图、熔点-组成图、溶解度-组成图

\section{(II-3-1) 沸点-组成图 $(T-x$ 图)}

典型实验: 挥发性二组分系统 $T-x$ 图的绘制 ${ }^{[9]}$; 完全互溶双液系/环己烷-乙醇体系沸点-组成图的 绘制 $[8,10]$

仪器设备：蒸馏装置 阿贝折射仪

实验建议: (1) 能够对温度计进行露茎校正; (2) 能够绘制 $T-x$ 图并指明系统的组成、恒沸混合 物组成和最低恒沸点; (3) 能够用 $T-x$ 图说明蒸馏、精馏的原理及其应用。

\section{(II-3-2) 熔点-组成图}

典型实验: 二组分简单共熔系统相图的绘制 $(\mathrm{Pb}-\mathrm{Sn} / \mathrm{Sn}-\mathrm{Bi} \text { 体系 })^{[9,11,13]}$; 二组分固-液相图的测绘 ${ }^{[15]}$

仪器设备: 电炉(加热炉、保温炉) 微分示差扫描量热仪 
实验建议: (1) 能够根据热分析法(步冷曲线法)、微分示差扫描量热法的数据绘制相图; (2) 能 够说明相图中各部分的含义; (3) 能够根据相图预测系统组成和变化规律; (4) 能够处理步冷过程中 的过冷现象和氧化现象。

\section{(II-3-3) 溶解度-组成图}

典型实验: 三液系(三氯甲烷-醋酸-水)相图的绘制 ${ }^{[9]}$; 三组分体系等温相图的绘制(苯-醋酸-水, 环已烷-乙醇-水) ${ }^{[8]}$

仪器设备：具塞雉形瓶 酸碱式滴定管 移液管 分液漏斗

实验建议：(1) 说明实验绘制三液系相图的原理; (2) 能够绘制等边三角形相图; (3) 能够对三 元相图进行分析说明。

\section{(II-4) 热分析}

典型实验: 差热分析 ${ }^{[8,10-13]} ; \mathrm{CuSO}_{4} \cdot 5 \mathrm{H}_{2} \mathrm{O}$ 在空气气氛中脱水过程的差热分析(DTA)测定 ${ }^{[9]}$; 差 热-热重分析及应用 ${ }^{[11]}$; TG/DSC-MS联用技术测定 $\mathrm{CaC}_{2} \mathrm{O}_{4} \cdot \mathrm{H}_{2} \mathrm{O}$ 热分解过程及动力学计算 ${ }^{[11]}$

仪器设备：差热分析仪(DTA) 示差扫描量热仪(DSC)

实验建议: (1) 说明差热分析的原理和参比物的作用; (2) 能够说明热分析谱图变化所对应的反 应或过程; (3) 说明利用DTA进行定性定量分析的原理; (4) 说明通过DTA测量热力学性质的原理; (5) 能够拓展DTA的应用。

\section{(III-1) 反应速率/速率常数/半衰期 \\ (III-1-1) 均相反应}

\section{(III) 动力学性质}

典型实验: 蔗糖水解反应速率常数的测 ${ }^{{ }^{2}}{ }^{9-13]}$; 乙酸乙酯㿝化反应速率常数的测定 ${ }^{[8-13]}$; 丙酮碘 化反应速率常数及活化能的测定 (复杂反应、反应机理) ${ }^{[8-10]}$; 比色法研究甲基紫反应动力学 ${ }^{[8]}$; 核磁 共振法测定水溶液中反应的平衡常数及反应速率常数 ${ }^{[9]}$; 弛豫法测定铬酸根-重铬酸根离子反应的速 率常数 ${ }^{[15]}$

仪器设备: 旋光仪 电导率仪 分光光度计 核磁共振仪 精密酸度计

实验建议: (1) 能够说明采用物理量测量浓度的优势与不足; (2) 能够采用作图法确定速率常 数; (3) 能够辨别准级数反应; (4) 能够通过实验验证反应机理; (5) 能够说明弛豫法测量对峙反应 速率常数的原理。

\section{(III-1-2) 多相反应/催化剂活性}

典型实验：量气法测定过氧化氢催化分解反应速率常数 ${ }^{[13]}$; 过氧化氢催化分解反应动力学 ${ }^{[10]}$; 催化剂活性的测定 ${ }^{[11]}$; 乙醇脱水制乙烯反应动力学常数的测定 ${ }^{[8]}$; 流动法测定 $\gamma-\mathrm{Al}_{2} \mathrm{O}_{3}$ 小球催化剂乙 醇脱水的催化性能 $[9]$

仪器设备: 量气管 平衡管 管式炉 *固定床反应装置 气体流量计 气相色谱仪

实验建议: (1) 能够准确控制和测量气体流速; (2) 能够说明表征催化剂活性的主要参数; (3) 能 够说明催化剂活性测量原理和方法; (4) 能够制备负载型催化剂。

\section{(III-1-3) 光催化反应}

典型实验: 纳米 $\mathrm{TiO}_{2}$ 光催化降解甲基橙 ${ }^{[9]}$; 纳米 $\mathrm{TiO}_{2}$ 的制备、光催化活性评价及催化动力学研 究 $^{[13]}$

仪器设备: 光化学反应装置 分光光度计

实验建议: (1) 能够说明光催化降解污染物的原理及其重要意义; (2) 能够说明提高光化学反应 效率的方法; (3) 能够辨析光化学反应和热化学反应的不同。 


\section{(III-2) 反应级数}

典型实验: 丙酮碘化反应速率常数及活化能的测定 ${ }^{[8]}$

仪器设备: 分光光度计 量气管

实验建议: (1) 能够说明采用孤立法、尝试法、作图法确定反应级数的原理; (2) 能够确定反应 的速率方程; (3) 能够分析准级数反应的可能性及其影响; (4) 能够说明反应级数测量对推测反应机 理的意义。

\section{(III-3) 活化能}

典型实验: 蔗糖水解反应速率常数的测定 ${ }^{[9-13]}$; 丙酮碘化反应速率常数及活化能的测定 ${ }^{[8]}$; 乙酸 乙酯㿝化反应速率常数和活化能的测定 ${ }^{[10]}$; 过二硫酸铵与碘化钾反应速率与活化能的测定 ${ }^{[10]}$

仪器设备：同(III-1-1)

实验建议: (1) 能够正确使用Arrhenius图确定反应活化能; (2) 能够说明影响活化能测量的因素。

\section{(III-4) 米氏常数}

典型实验：蔗糖酶米氏常数的测定 ${ }^{[8]}$; 分光光度法测定蔗糖酶的米氏常数 ${ }^{[15]}$

仪器设备: 分光光度计 旋光仪

实验建议: (1) 能够说明Michaelis-Menten机理并导出其动力学方程; (2) 通过线性化方法确定 米氏常数。

\section{(III-5) 自催化}

典型实验：B-Z振荡反应 ${ }^{[8,13]}$

仪器设备: 电化学工作站 参比电极 Pt电极

实验建议: (1) 能够说明振荡反应发生的机理; (2) 能够说明振荡反应对非平衡态、非线性热力 学研究的意义; (3) 能够说明B-Z振荡反应的影响因素。

\section{(III-6) 反应机理/反应分子数的理论研究}

典型实验: $\mathrm{H} \cdot+\mathrm{CH}_{4}=\mathrm{H}_{2}+\mathrm{CH}_{3}$. 和D $+\mathrm{CH}_{4}=\mathrm{HD}+\mathrm{CH}_{3}$. 反应动力学参数的计算 ${ }^{[9]}$; 计算机模拟 基元反应 ${ }^{[11,15]} ;$ Diels-Alder反应速率常数的计算 ${ }^{[11]}$

仪器设备：计算机 量化计算软件(VASP, Gauss, Materials Studio)

实验建议: (1) 能够根据不同的化学反应选取不同的理论计算模型; (2) 能够分析计算过程中各 参数的含义及对计算结果的影响; (3) 能够基于量子化学计算从微观层面说明反应过程, 形成从微 观角度理解反应本质的意识; (4) 能够通过量子化学计算揭示反应通道和决速步骤; (5) 能够利用分 子反应碰撞理论和过渡态理论描述微观反应过程。

\section{(III-7) 热力学量的理论计算}

典型实验: 甲烷生成热和燃烧热的理论计算 ${ }^{[9]}$

仪器设备：计算机 量化计算软件(GaussView, Gaussian)

实验建议: (1) 能够创建与修改分子结构模型并进行量子化学计算; (2) 能够基于理想气体模型, 以统计热力学方法计算生成热、燃烧热等热力学量; (3) 能够分析不同量子化学方法的计算精度和 计算代价; (4) 能够根据分子结构的复杂性和问题属性正确选择计算方法; (5) 能够说明理论计算的 重要意义。

\section{(IV-1) 电导率}

\section{(IV) 电化学性质}

典型实验: 电导的测定及其应用 ${ }^{[9]}$; 电导法测定弱电解质的解离平衡常数 ${ }^{[10]}$; 电导法测定难溶 盐的溶解度 ${ }^{[13]}$; 电导法测定乙酸乙酯㿝化反应的速率常数 ${ }^{[15]}$; 电导法测定水溶性表面活性剂的临界 
胶束浓度 ${ }^{[15]}$; 电导滴定法测定食醋中总酸含量 ${ }^{[28]}$

仪器设备: 电导率仪 电导电极

实验建议: (1) 能够说明电导电极选择的依据; (2) 能够说明电导测量在工业、农业、医药、食 品、科研和环保等领域的应用; (3) 能够说明电导测量在在国家标准认证(QS)、食品安全认证体系 (HACCP)中的重要意义。

\section{(IV-2) 离子迁移数}

典型实验：希托夫法测定离子迁移数 ${ }^{[11,13]}$; 离子迁移数的测定一一界面法 ${ }^{[11,12]}$

仪器设备: 希托夫管 恒压电源 迁移管

实验建议: (1) 能够说明希托夫法、界面移动法、电动势法、分光光度法、*交流阻抗法、*计时 电流法测量离子迁移数的原理并比较其优劣; (2) 能够分析其他传质过程对测量的影响及优化办法。

\section{(IV-3) 电池电动势/电动势温度系数/热力学函数}

典型实验: 电动势法测定热力学函数 ${ }^{[8]}$; 原电池电动势的测定及原电池热力学 ${ }^{[10]}$; 电池电动势 法测定氯化银的溶度积 ${ }^{[13]}$

仪器设备: 电势差计/恒电位仪 电化学工作站 电池 恒温槽

实验建议: (1) 能够制作电极并合理组装电池; (2) 能够说明采用电势差计测量电动势的原理; (3) 能够说明消除电池中不可逆过程的原理和方法; (4) 能够说明通过测量电动势及其温度系数测量 热力学函数的原理; (5) 能够说明电化学方法测量热力学函数的优劣及适用条件。

\section{(IV-4) 电极电势}

典型实验：电极电势的测定 ${ }^{[11]}$; 难溶盐 $\mathrm{AgCl}$ 溶度积的测定 ${ }^{[11]}$; 测定溶液的 $\mathrm{pH}$ 值 ${ }^{[11]}$

仪器设备: 电位差计 电化学工作站 参比电极 $\mathrm{pH}$ 计 标准氢电极

实验建议: (1) 能够说明参比电极的主要种类、特征和选用原则; (2) 能够说明可逆电池组装时 应注意的问题; (3) 能够说明消除或者减小液体接界电势的原理和方法。

\section{(IV-5) 电势-pH图(Pourbaix图)}

典型实验: 电势 $-\mathrm{pH}$ 曲线的测定 ${ }^{[8,9,11,12]}$

仪器设备: 同(IV-4)

实验建议: (1) 能够说明图形化方法的意义; (2) 能够说明电势- $\mathrm{pH}$ 图中各线、区、点的物理意 义; (3) 能够根据电势- $\mathrm{pH}$ 图确定稳定物种以及发生电化学反应的可能性; (4) 能够用电势- $\mathrm{pH}$ 图说 明腐蚀防护原理。

\section{(IV-6) 极化曲线/超电势 \\ (IV-6-1) 极化曲线}

典型实验: 极化曲线的测定 ${ }^{[11]}$; 极化曲线和氢超电势的测定 ${ }^{[10]}$; 碳钢在碳酸铵溶液中的阳极和 阴极极化曲线 $[11]$

仪器设备: 恒电位仪/电化学工作站 三电极电解池 参比电极 辅助电极

实验建议: (1) 能够说明准稳态恒电势法测定金属极化曲线的基本原理; (2) 能够利用线性化方 法得出Tafel关系。

\section{(IV-6-2) 氢超电势}

典型实验: 极化曲线和氢超电势的测定 ${ }^{[10]}$; 氢超电势的测定 ${ }^{[8,15]}$

仪器设备: 恒电位仪/电化学工作站 三电极电解池 参比电极 辅助电极 氢气发生器

实验建议: (1) 能够利用线性化方法得出 Tafel公式并说明斜率和截距的物理意义; (2) 能够说明 影响超电势的主要因素; (3) 能够用一分为二的观点分析氢超电势的应用。 


\section{(IV-6-3) 金属针化曲线}

典型实验: 镍在硫酸溶液中的钝化行为 ${ }^{[8]}$; 线性电位扫描法测定镍在硫酸溶液中的钝化行为 ${ }^{[15]}$; 金属铁的针化与防腐行为评价测定及应用 ${ }^{[13]}$

仪器设备: 同(IV-6-1)

实验建议: (1) 能够说明钝化曲线各个阶段所对应的表面过程; (2) 能够说明钝化的种类, 产生 钝化的原因和影响针化的因素; (3) 能够概述针化在增强金属腐蚀防护性能领域的应用。

\section{(IV-7) 金属电镀}

典型实验: 金属的电镀实验 ${ }^{[28]}$

仪器设备: 电镀槽 直流稳压电源

实验建议: (1) 能够说明电镀、合金镀、复合镀、塑料电镀的原理; (2) 能够说明整平剂、光亮 剂等电镀添加剂的作用原理; (3) 能够说明评价电镀液分散能力和电流效率的方法; (4) 能够说明电 镀的重要应用。

\section{(IV-8) 金属腐蚀与防护}

典型实验: 金属铁的针化与防腐行为评价测定及应用 ${ }^{[13]}$; 缓蚀剂、阴极保护对碳钢腐蚀防护效 果的测定 ${ }^{[28]}$

仪器设备: 电化学工作站 电导率仪

实验建议: (1) 能够说明金属腐蚀基本理论及防止金属腐蚀的主要原理和技术; (2) 能够说明采 用失重法、析气法、极化曲线法测量金属腐蚀速率的原理; (3) 能够说明介质、介质浓度等对金属腐 蚀速率的影响; (4) 能够分析不同材料发生腐蚀的原因; (5) 能够说明防止金属腐蚀的原理和方法及 腐蚀防护对国家安全发展的重要意义。

\section{(V-1) 液体表面张力}

\section{(V) 表面与胶体性质}

典型实验：最大泡压法测定溶液的表面张力 ${ }^{[10]}$; 吊环法测定溶液的表面张 力 $^{[30]}$; 毛细管法 ${ }^{[30] 、}$ 滴重法测定液体的表面张力 ${ }^{[31]}$

仪器设备: 表面张力仪

实验建议: (1) 能够说明毛细管法、最大气泡压力法、DuNouy环法、Wilhelmy吊片法、旋转法、 滴重法、滴体积法等测量表面张力的原理; (2) 能够从微观和分子间作用力的角度说明表面张力产 生的原因; (3) 能够说明表面张力的影响因素; (4) 能够说明表面张力与溶液表面吸附的关系。

\section{(V-2) 比表面积/孔径/孔径分布/孔体积/孔隙度/吸附等温线}

典型实验：BET容量法测定固体比表面积 ${ }^{[10,15]}$; 溶液吸附法测定固体比表面积 ${ }^{[13]}$; 固体比表面 积的测定 ${ }^{[9]}$; 材料孔结构测定 ${ }^{[32]}$

仪器设备: 全自动物理吸附仪 压录仪 分光光度计

实验建议：(1) 能够说明BET法、Langmuir法、溶液吸附法、压永法测量固体比表面积的原理; （2）能够分析吸附等温线得出孔体积、孔径分布等物理量; (3) 能够说明增加物质比表面积的主要方 法及其原理; (4) 能够说明吸附剂再生的原理和方法; (5) 能够说明采用双电层电容测量电极(电催 化剂)比表面积的原理。

\section{(V-3) 吸附量}

\section{(V-3-1) 气-固界面}

典型实验：BET容量法测定固体的比表面积 $[10,15]$

仪器设备: 吸附仪 吸附天平 气相色谱仪

实验建议: (1) 能够说明容量法、重量法、动态法(色谱法)测量吸附量的原理; (2) 能够根据测 
量要求选择适宜的测量方法; (3) 能够比较不同测量方法的优劣及其适用性; (4) 能够分析影响吸附 量测量的因素; (5) 能够说明增强吸附剂吸附能力的原理和方法。

\section{(V-3-2) 液-固界面}

典型实验：溶液吸附法测定固体物质比表面 ${ }^{[13]}$

仪器设备: 分光光度计

实验建议: (1) 能够说明溶液吸附法测量比表面积的原理; (2) 能够选择不同方法测量金属离子、 挥发性有机物、生物分子等的吸附量; (3) 能够分析影响吸附量测量的因素; (4) 能够设计吸附方案 处理重金属离子、有机挥发物等污染。

\section{(V-4) 溶液表面过剩浓度}

典型实验：溶液中的吸附作用和表面张力的测定 ${ }^{[9]}$

仪器设备: 表面张力仪 (最大气泡压力法)

实验建议: (1) 能够说明测量溶液表面过剩量的原理; (2) 能够说明表面过剩量与表面张力变化 的关系; (3) 能够从微观和分子间作用力的角度说明表面过剩量随浓度的变化趋势。

\section{(V-5) 胶体电泳速率/淌度/电动电势}

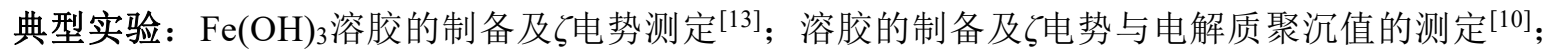
胶体电泳速度的测定 ${ }^{[9]}$; 电渗 电泳实验 ${ }^{[15]}$

仪器设备: 电泳仪 $*$ 显微电泳仪

实验建议: (1) 能够制备和纯化胶体; (2) 能够分析影响胶体粒子电泳速度的因素; (3) 能够评 估胶体粒子的带电性质, 说明改变胶体粒子带电性质的方法及其原理; (4) 能够设计纳米粒子制备 方法, 说明纳米材料在疾病检测、高性能催化剂开发等方面的应用。

\section{(V-6) 临界胶束浓度(CMC)}

典型实验: 表面活性剂的类型鉴别及临界胶束浓度 $\mathrm{CMC}$ 的测定 ${ }^{[10]}$; 电导法测定水溶性表面活性 剂的临界胶束浓度 ${ }^{[15]}$; 水溶性表面活性剂临界胶束浓度的测定 ${ }^{[8]}$

仪器设备: 表面张力仪 电导率仪

实验建议: (1) 能够说明表面张力法、电导法、染料法、增溶法、渗透压法、苂光法、pH法、 流变法、浊度法、*光散射法测量 $\mathrm{CMC}$ 的原理; (2) 能够说明影响临界胶束浓度的因素; (3) 能够说 明临界胶束浓度测量的意义与应用。

\section{(V-7) 接触角/润湿角}

典型实验：固-液界面接触角的测量 ${ }^{[8]}$; 碳酸钙纳米颗粒的制备及表面疏水化 ${ }^{[8]}$

仪器设备：接触角测量仪

实验建议: (1) 能够说明影像分析法、力测量法、*插板法、*透过测量法测量接触角的原理; （2）能够分析影响接触角的因素; (3) 能够运用接触角测量正确评估固液界面性质; (4) 能够设计方 案解决玻璃防雾、固体表面疏水处理等实际问题。

\section{(V-8) 流变性质}

典型实验: 非牛顿型流体流变曲线的绘制[8,15]

仪器设备: 旋转黏度计

实验建议: (1) 能够说明旋转黏度计测量原理; (2) 能够根据流变曲线判定流体特性; (3) 能够 说明流体结构组成与流变参数的关系; (4) 能够说明流体特性研究在设计沥青、润滑油等产品, 评价 品质中的应用。

\section{(V-9) 粒度/粒径}

典型实验: 粒度测定 ${ }^{[11]}$

仪器设备: 激光粒度仪 超声波清洗器 
实验建议: (1) 能够说明粒度测量的原理; (2) 能够说明影响粒度测量的因素; (3) 能够列举其 他测量粒度或者粒径的方法并比较其优劣。

\section{(VI) 分子的结构与性质}

\section{(VI-1) 磁化率}

典型实验: 磁化率的测定 $[9,10,12,13,15]$

仪器设备: 古埃磁天平 特斯拉计

实验建议: (1) 能够说明古埃(Gouy)法测量磁化率的原理; (2) 能够使用标准样品(莫尔盐)进行 仪器标定; (3) 能够计算化合物的永久磁矩和未成对电子数; (4) 能够根据未成对电子数判断配键类 型; (5) 能够分析影响实验测量准确度的因素。

\section{(VI-2) 偶极矩}

典型实验：偶极矩测量 ${ }^{[8-10,12,13]}$; 溶液法测定极性分子的偶极矩 ${ }^{[15]}$

仪器设备: 小电容测量仪(介电常数测量仪) 阿贝折射仪 偶极矩仪 密度管

实验建议: (1) 能够说明采用介电常数法(溶液法、气相法、电桥法、共振法和拍频法)测量分子 偶极矩的实验原理; (2) 能够对偶极矩测量误差进行分析; (3) 能够说明测量偶极矩对研究分子结构 的意义。

\section{(VI-3) 配位数/配合物组成}

典型实验：配合物组成及不稳定常数的测定 ${ }^{[13]}$; 磺基水杨酸合铁配合物组成和稳定常数的测定 ${ }^{[7]}$

仪器设备: 分光光度计

实验建议: (1) 能够说明分光光度法测量配位数的原理并分析产生实验误差的原因; (2) 能够根 据配合物特征选取适宜的测量方法。

\section{(VI-4) 晶体结构参数}

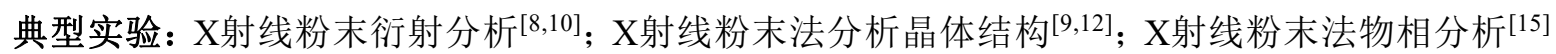

仪器设备: X射线衍射仪

实验建议: (1) 能够说明粉末法(照相法/德拜法、衍射仪法)、单晶法(劳厄Laue)法、周转晶体法、 四元衍射仪法测量晶体参数的原理; (2) 能够说明腐蚀性法、激光定向法、X射线衍射法确定晶面取 向的原理; (3) 能够确定各晶面指标并计算晶胞常数; (4) 能够计算晶胞中所含原子、分子或离子的 数目；(5) 能够使用PDF卡片及检索工具对样品进行物相分析；(6) 能够分析实验误差。

\section{(VI-5) 分子微观结构参数}

典型实验：红外光谱测定 $\mathrm{HCl}$ 的结构参数 ${ }^{[8,9]}$; 红外光谱法测定简单分子的结构参数 ${ }^{[15]}$

仪器设备: 红外光谱仪 气体样品池

实验建议: (1) 能够说明分子光谱产生的原理; (2) 能够对红外光谱进行解析并计算解离能、转 动惯量、力常数和平衡键距等分子结构参数; (3) 能够分析实验误差。

\section{4 物理化学实验教学建议}

物理化学实验中涉及大量中小型仪器设备的使用, 主要包括《化学类专业化学实验教学建议内 容》[3] “V仪器设备与软件” 中的1-6以及9 (1)所列仪器设备。这些仪器设备的原理和使用相对复杂, 又不宜占用大量实验时间进行学习和练习, 建议各高校通过建设线上资源, 使用操作视频、原理讲 解视频、虚拟仿真实验等教学资源, 推动学生以自主学习的方式提前掌握并通过考核。另外, 由于 物理化学实验的原理相对复杂, 临时讲解的效果难以保证, 也推荐利用线上资源, 安排学生通过课 外自主学习的方式提前掌握。即物理化学实验应该走在信息化建设的前列, 充分利用计算机、软件 和信息化平台等手段, 利用各类教学资源和手段, 提升学生的自主学习能力和信息化素养, 保证实 
验教学效果。

此外, 为了增强学生分析和解决问题的能力、创新能力, 建议增加物理化学设计实验、才队实 验，增加研讨环节，并引导学生进行原理和应用的拓展。

致谢: 感谢吉林师范大学赵丽娜教授、浙江工业大学唐浩东副教授、同济大学王晓岗高级工程师、厦门大学吴平平 老师反馈修改意见。吴平平老师还制作了精美的附图(见文末)，特别致谢。

\section{参 考 文 献}

[1] 教育部高等学校教学指导委员会. 普通高等学校本科专业类教学质量国家标准(上). 北京: 高等教育出版社, 2018.

[2] 张树永, 侯文华, 刀国旺. 大学化学, 2017, 32 (9), 1.

[3] 2013-2017年教育部高等学校化学类专业教学指导委员会. 大学化学, 2017, 32 (8), 1 .

[4] 张树永, 朱亚先, 张剑荣. 大学化学, 2018, 33 (10), 1.

[5] 张树永. 中国大学教学, 2015, No. 3, 55 .

[6] Adnerson, L. W.; Krathwohl, D. R.; Airasian, P. W.; Cruikshank, K. A.; Mayer, R. E.; Pintrich, P. R.; Raths, J.; Wittrock, M. C. 布鲁姆教育目标分 类学(修订版). 蒋小平, 张美琴, 罗晶晶, 译. 北京: 外语教学与研究出版社, 2009.

[7] 范勇, 屈学俭, 徐家宁. 基础化学实验(无机化学实验分册). 第2版. 北京: 高等教育出版社, 2017.

[8] 吉林大学, 朱万春, 张国艳, 李克昌, 徐家宁. 基础化学实验—物理化学实验分册. 第2版. 北京: 高等教育出版社, 2017.

[9] 邱金恒, 孙尔康, 吴强. 物理化学实验. 北京: 高等教育出版社, 2010.

[10] 天津大学物理化学教研室, 冯霞, 朱莉娜, 朱荣娇. 物理化学实验. 北京: 高等教育出版社, 2015.

[11] 山东大学, 山东师范大学等校, 合编. 宋淑娥, 主编. 基础化学实验(III)物理化学实验. 第3版. 北京: 化学工业出版社, 2019.

[12] 贺德华, 麻英, 张连庆. 基础物理化学实验. 北京: 高等教育出版社, 2008.

[13] 贾能勤, 王秀英, 黄楚森. 物理化学实验. 北京: 高等教育出版社, 2017.

[14] 宋光泉. 大学通用化学实验技术(上册). 北京: 高等教育出版社, 2009.

[15] 复旦大学. 物理化学实验. 第3版. 北京: 高等教育出版社, 2004.

[16] 张锁秦, 张广良, 宋志光, 徐家宁. 基础化学实验(有机化学实验分册). 第2版. 北京: 高等教育出版社, 2017.

[17] 田月兰. 高分子科学综合实验教程. 北京: 化学工业出版社, 2019.

[18] 北京师范大学. 无机化学实验. 第4版. 北京: 高等教育出版社, 2014.

[19] 侯炜, 戴莹䒯. 物理化学实验. 北京: 北京理工大学出版社, 2016.

[20] 高峰, 任福正, 翁伟宇, 陈彦佐, 景秋芳. 药剂学实验. 上海: 华东理工大学出版社, 2015.

[21] 陈金榜. 物理化学学报, 1993, 9 (2), 121.

[22] 吴洪达, 叶旭. 物理化学实验. 武汉: 华中科技大学出版社, 2017.

[23] 武汉大学化学与分子科学学院实验中心. 物理化学实验. 武汉: 武汉大学出版社, 2012.

[24] 周建萍. 高分子材料与工程专业实验. 北京: 北京航空航天大学出版社, 2018: 10 .

[25] 张秀成, 刘冰, 王玉峰. 应用物理化学实验. 哈尔滨: 东北林业大学出版社, 2009.

[26] 曹波, 陈昌国. 现代基础化学实验. 重庆: 重庆大学出版社, 2010.

[27] 夏海涛. 物理化学实验. 南京: 南京大学出版社, 2019.

[28] 岳可芬. 基础化学实验III 物理化学实验. 北京: 科学出版社, 2012.

[29] 周井炎. 基础化学实验下. 武汉: 华中科技大学出版社, 2008.

[30] 李平舟. 综合设计物理实验. 西安: 西安电子科技大学出版社, 2012.

[31] 咸春颖, 沈丽, 张帅. 物理化学实验. 上海: 东华大学出版社, 2018.

[32] 罗永勤，高云琴. 无机非金属材料实验. 北京: 治金工业出版社, 2018. 
附图:

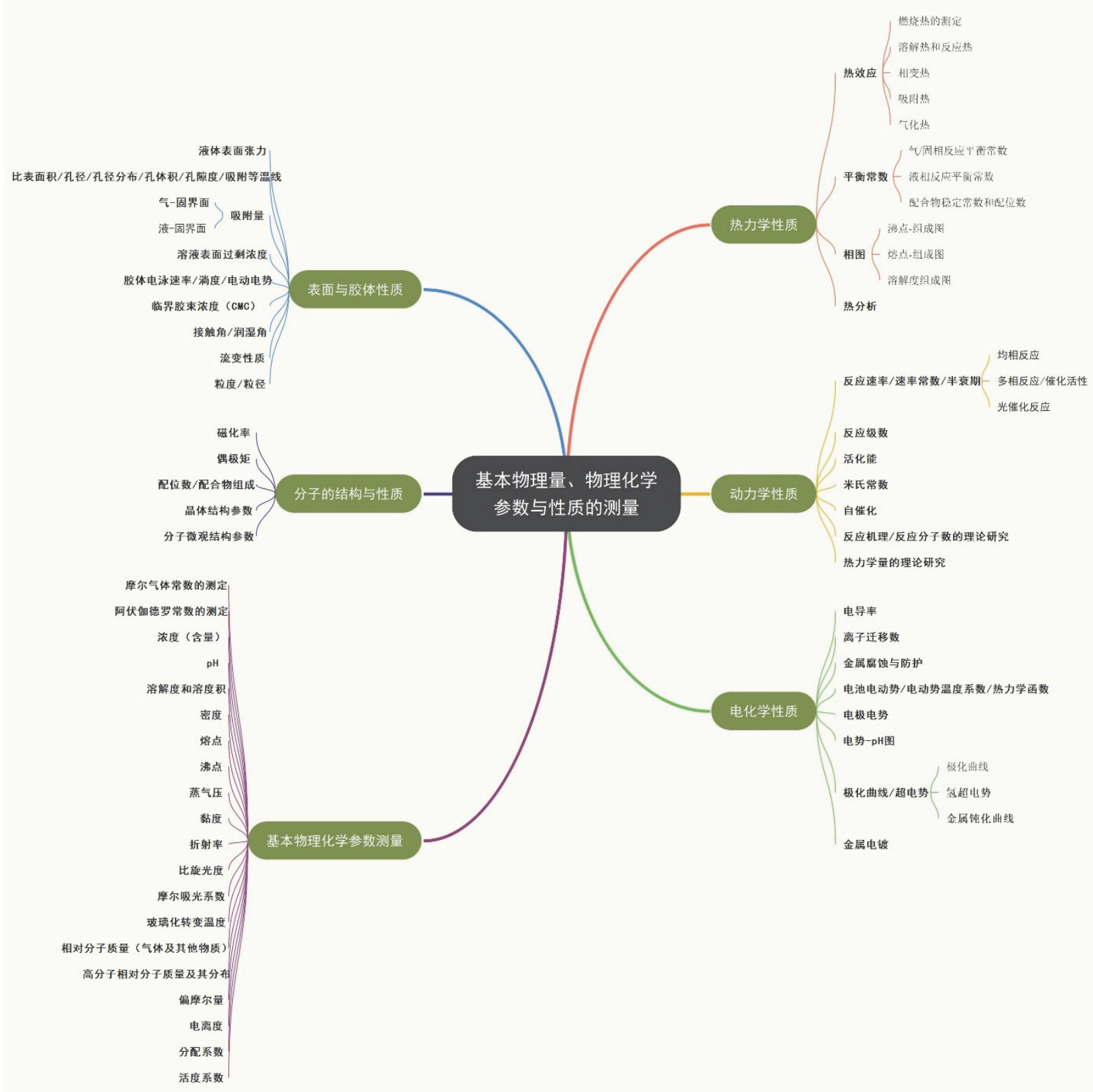

